\title{
THE WIND TEMPERATURE AND C/He AND O/He RATIOS OF THE WC10 CENTRAL STAR CPD $-56^{\circ} 8032$
}

\author{
M. J. BARLOW and P. J. STOREY \\ Department of Physics $\&$ Astronomy, University College London, \\ Gower Street, London WC1E 6BT, U.K.
}

We have analysed absolutely calibrated $3500-8900 \AA$ AAT spectrophotometry of the WC10 central star CPD- $56^{\circ} 8032$. After dereddening by $\mathrm{E}(\mathrm{B}-\mathrm{V})=0.68$, the few line-free portions of the stellar continuum are well fitted by the $18000 \mathrm{~K}$ blackbody which corresponds to the nebular H I Zanstra temperature. The Wolf-Rayet wind terminal velocity is sufficiently low $\left(210-230 \mathrm{~km} \mathrm{~s}^{-1}\right)$ that it is possible to resolve virtually every fine structure component of every multiplet of C II, C III and O I listed by the NBS Multiplet Tables for this wavelength region.

Many of the observed C II recombination lines in the spectrum of CPD- $56^{\circ} 8032$ are due to the process of low temperature dielectronic recombination. They arise from the radiative decay, and subsequent cascade, of strongly autoionizing resonance states near the ionization limit of $\mathrm{C}+$. The populations of such resonance states are determined by the balance between autoionization and radiative decay. Autoionization usually dominates, and the populations are then given by the Saha equation. The measured fluxes of such lines may therefore be used to infer the electron temperature. We have identified four such C II lines in the spectrum of CPD$56^{\circ} 8032 ; \lambda \lambda 4621,4961,5113,8798$. Two of the transitions $(\lambda 4621$ and $\lambda 5113)$ are between states residing entirely in the continuum and should therefore be thought of as resonance features in the free-free emission associated with $\mathrm{C} 2+$, rather than as recombination lines. Our fit to these four dielectronic recombination lines of C II yields an emission measure of $9.0 \pm 0.1 \times 10^{13} \mathrm{~cm}^{-5}$ for $\mathrm{C} 2+$, along with a wind temperature of $12800 \mathrm{~K}$, which can be used for a recombination line abundance analysis of the other ions that are present in the wind. The stronger lines of He I (no lines of hydrogen are emitted by the wind) all show $\mathrm{P}$ Cygni profiles due to self-absorption, but the weak He I lines at $4713 \AA$ and $5047 \AA$ both show Gaussian profiles and are unblended. The dereddened fluxes measured for these lines have been used with high density recombination coefficients to derive a $\mathrm{He}+$ emission measure (I/Q) equal to $5.82 \pm 0.3 \times 10^{14} \mathrm{~cm}^{-5}$. We have determined the abundance of $\mathrm{O} 2+$ in the wind from the O II recombination lines at $\lambda \lambda 4489.77,4491.54$ and $\lambda \lambda 4890.93,4906.98$ We derive an $\mathrm{O} 2+$ emission measure $(\mathrm{I} / \mathrm{Q})$ of $4.1 \pm 2.1 \times 10^{13} \mathrm{~cm}^{-5}$. C3+ was found not to make a significant contribution to the total abundance of carbon.

The $\mathrm{C} / \mathrm{He}$ ratio of 0.15 by number is comparable to those found for Population I WC Wolf-Rayet stars, but the $\mathrm{O} / \mathrm{He}$ ratio of 0.07 is significantly higher than is believed applicable for Population I WC stars. This may not be surprising in view of the completely different nuclear-burning histories of massive WC stars and PN WC stars. The relatively high oxygen abundance that is found implies that when CPD$56^{\circ} 8032$ evolves to higher effective temperatures it will become an O VI emission line central star and, eventually, a low luminosity pulsating PG1159-035 star. 